\title{
POLYNOMIAL DENSITY IN BERS SPACES
}

\author{
JACOB BURBEA ${ }^{1}$
}

\begin{abstract}
Let $D$ be a bounded Jordan domain such that $\iint_{D} \lambda_{D}^{2-q} d x d y$ $<\infty$ for $q>1$. Here $\lambda_{D}(z)$ is the Poincare metric for $D$. Define $A_{q}^{p}(D)$, the Bers space, to be the Fréchet space of holomorphic functions $f$ on $D$, such that $\|f\|_{q, p}^{p}=\iint_{D} \lambda_{D}^{2-q p}|f|^{p} d x d y$ is finite, $0<p<\infty, q p>1$. It is well known that the polynomials are dense in $A_{q}^{p}(D)$ for $q p>2$. We show that they are dense in $A_{q}^{P}(D)$ for $q p>1$ irrespective whether the boundary of $D$ is rectifiable or not.
\end{abstract}

1. Introduction. Let $D$ be a bounded Jordan domain such that

$$
\iint_{D} \lambda_{D}^{2-q}(z) d x d y<\infty
$$

where $\lambda_{D}(z)$ is the Poincare metric for $D$. Since $\lambda_{D}^{-1}(z) \leqslant \sqrt{A / \pi}$, where $A$ is the area of $D$, it follows that (1.1) obviously holds for all $q \geqslant 2$. Hence we can assume that (1.1) holds for all $q>q_{0}$ where $1 \leqslant q_{0}<2$ (the case $q \leqslant 1$ is, of course, trivial). Let $\phi: D \rightarrow U$ be a Riemann mapping of $D$ onto $U$, the unit disc, and let $\psi=\phi^{-1}$. Then

$$
\iint_{D} \lambda_{D}^{2-q}(z) d x d y=\iint_{U}\left(1-|w|^{2}\right)^{q-2}\left|\psi^{\prime}(w)\right|^{q} d u d v .
$$

A well-known inequality due to Hardy and Littlewood [5] states that for $q>1, r>0$,

$$
\left\{\iint_{U}\left(1-|w|^{2}\right)^{q-2}|f(w)|^{r} d u d v\right\}^{1 / r} \leqslant c\|f\|_{r / q},
$$

where $c$ is a constant depending on $q$ and $r$ and \|\|$_{r / q}$ is the $H^{r / q}$ norm. Here $H^{r / q}=H^{r / q}(U)$ is the $r / q$ Hardy class.

Since for Jordan domains the rectifiability of the boundary is equivalent to $\psi^{\prime} \in H^{1}(U)[3$, p. 44], it follows from (1.2) and (1.3) that

Proposition 1. Let $D$ be a bounded rectifiable Jordan domain. Then (1.1) holds for all $q>1$.

However, the property that (1.1) holds for all $q>1$ is not characteristic to rectifiable domains. In fact,

Received by the editors December 15, 1975.

AMS (MOS) subject classifications (1970). Primary 30A82; Secondary 30A58, 41A10.

Key words and phrases. Bers spaces, Poincare metric, polynomial density.

'This work is supported by U. S. Army Grant 2065. 
Proposition 2. There is a domain $D$, bounded by a nonrectifiable Jordan curve, such that (1.1) holds for all $q>1$.

Proof. According to Hedberg [6] there is such a domain $D$ with

$$
\iint_{D}\left(1-|\phi(z)|^{2}\right)^{q-2} d x d y<\infty
$$

for all $q>1$. Since (1.1) holds for all $q \geqslant 2$ we can assume that $1<q<2$. An application of Hölder's inequality yields

$$
\begin{aligned}
\iint_{U}(1 & \left.-|w|^{2}\right)^{q-2}\left|\psi^{\prime}(w)\right|^{q} d u d v \\
& \leqslant\left\{\iint_{U}\left(1-|w|^{2}\right)^{q-2}\left|\psi^{\prime}(w)\right|^{2} d u d v\right\}^{q / 2} \cdot\left(\frac{\pi}{q-1}\right)^{1-q / 2} .
\end{aligned}
$$

The proposition now follows from (1.4).

We shall only consider those domains $D$ such that (1.1) holds for all $q>1$, those $D$ for which (1.1) holds for $q>q_{0}>1$ will be considered elsewhere. For $0<p<\infty$ and $q p>1$ we define $A_{q}^{p}(D)$, the Bers space, as the Fréchet space of holomorphic functions $f(z)$ on $D$, "normed" by

$$
\|f\|_{q, p}=\left\{\iint_{D} \lambda_{D}^{2-q p}(z)|f(z)|^{p} d x d y\right\}^{1 / p} .
$$

Clearly $A_{q}^{p}(D)$ is a Banach space for $1 \leqslant p<\infty, q p>1$, and it is a Fréchet space for $0<p<1, q p>1$, with the usual metric $d(f, g)=\|f-g\|_{q, p}^{p}$, $f, g \in A_{q}^{p}(D)$. Also, since $D$ is bounded, the assumption about (1.1) implies that the polynomials belong to $A_{q}^{p}(D)$ for all $0<p<\infty$ and $q p>1$.

The question of polynomial density in $A_{q}^{1}(D)$ has been considered by various authors. For $q \geqslant 2$, Bers [2] and Knopp [7] proved that the polynomials are dense in $A_{q}^{1}(D)$ without any assumption on the mapping function $\psi$. Later Sheingorn [10] proved that the polynomials are dense in $A_{q}^{1}\left(U^{*}\right)$, $1<q<\infty$, where $U^{*}$ is a special Jordan domain introduced first by Earle and Marden [4] and used by Knopp [7] to prove his main lemma. Metzger [8] proved that if $\psi^{\prime} \in H^{1}(U)$ and $q>\frac{3}{2}$ then the polynomials are dense in $A_{q}^{1}(D)$. Recently, Metzger [9] was able to improve his result, and he actually showed that if $\psi^{\prime} \in H^{1}(U)$ then the polynomials are dense in $A_{q}^{1}(D)$ for all $q>1$. Our contribution in this paper is in showing that the polynomials are dense in $A_{q}^{1}(D)$ for all $q>1$ without any assumption on the boundary behavior of $\psi^{\prime}$, and, in view of Propositions 1 and 2, Metzger's results are obtained as a special case. In fact we will prove

THeOREM 1. Let $D$ be a bounded Jordan domain. Then the polynomials are dense in $A_{q}^{p}(D)$ for $0<p<\infty, q p>1$.

In order to prove this theorem we consider the space $\mathcal{H}_{q}^{p}(D)=A_{q / p}^{p}(D)$ instead of $A_{q}^{p}$. Therefore, $\mathcal{H}_{q}^{p}(D)$ is the Fréchet space of holomorphic functions $f(z)$ on $D$ normed by 


$$
\|f\|_{q, p}=\left\{\iint_{D} \lambda_{D}^{2-q}(z)|f(z)|^{p} d x d y\right\}^{1 / p},
$$

where $q>1,0<p<\infty$. Here $\|1\|_{q, 1}=\iint_{D} \lambda_{D}^{2-q}(z) d x d y<\infty$ for all $q>$ 1.

Using this notation, Theorem 1 can be restated as follows:

TheOREM 1'. Let $D$ be a bounded Jordan domain. Then the polynomials are dense in $\mathcal{H}_{q}^{p}(D)$ for $0<p<\infty, q>1$.

2. Auxiliary facts. In the case $q \geqslant 2$, Theorem $1^{\prime}$ was actually proved by Bers [2] although his result is stated for only the case $p=1$.

LEMMA 1 (BERS). Let $D$ be a bounded Jordan domain. Then the polynomials are dense in $\mathcal{H}_{q}^{p}(D)$ for $0<p<\infty, q \geqslant 2$.

The following lemma is by now standard.

LEMMA 2. The polynomials are dense in $\mathcal{H}_{q}^{p}(U)$ for $0<p<\infty, q>1$.

Using the Carathéodory-Walsh theorem [11, p. 36] we can show (see also [8], [10])

LemMa 3. Let $0<p<\infty, q>1$. The polynomials are dense in $\mathcal{F}_{q}^{p}(D)$ if and only if $\left(\phi^{\prime}\right)^{q / p}$ is in the $\mathcal{H}_{q}^{p}(D)$-closure of the polynomials.

The following technical lemma is needed for proving the main theorem.

LEMMA 4. Let $\alpha>0$ and $1<s<\infty$ such that

$$
(1-1 / \alpha) s=1-Q / 2, \quad Q>1 \text {. }
$$

If

$$
s(1+q-2 / \alpha)>1
$$

then

$$
\mathcal{F}_{Q}^{p s}(D) \subset \mathcal{H}_{q}^{p}(D), \quad q>1,
$$

the injection being cortinuous. If also

$$
s[q+4(1-1 / \alpha)]<3
$$

then $\left(\phi^{\prime}\right)^{q / p}$ is in $\mathcal{F}_{Q}^{p s}(D)$.

Proof. We have

$$
\|f\|_{q, p}^{p}=\iint_{D} \lambda_{D}^{2-2 / \alpha+2 / \alpha-q}|f|^{p} d x d y .
$$

An application of Hölder's inequality with $1<s<\infty$ and $s^{\prime}=s /(s-1)$ yields

$$
\begin{aligned}
\|f\|_{q, p}^{p} \leqslant & \left\{\iint_{D} \lambda_{D}^{2(1-1 / \alpha) s}|f|^{p s} d x d y\right\}^{1 / s} \\
& \times\left\{\iint_{D} \lambda_{D}^{2(1 / \alpha-q / 2) s /(s-1)} d x d y\right\}^{(s-1) / s}
\end{aligned}
$$


The first integral on the right-hand side is the $\mathcal{F}_{Q}^{p s}(D)$ norm, as $(2.1)$ shows. The second integral is finite if $2(1 / \alpha-q / 2) s /(s-1)<1$ which is exactly (2.2). Therefore, (2.3) is proved. We now show that $\left(\phi^{\prime}\right)^{q / p}$ is in $\mathcal{H}_{Q}^{p s}(D)$ under the above conditions. We, of course, can assume that $0 \in D$ and that $\phi(0)=0$. Now,

$$
\begin{aligned}
\left\|\left(\phi^{\prime}\right)^{q / p}\right\|_{Q, p s}^{p s} & =\iint_{D} \lambda_{D}^{2(1-1 / \alpha) s}\left|\phi^{\prime}(z)\right|^{q s} d x d y \\
& =\iint_{U}\left(1-|w|^{2}\right)^{2(1 / \alpha-1) s}\left|\psi^{\prime}(w)\right|^{2-s-s(1+q-2 / \alpha)} d u d v .
\end{aligned}
$$

Since $1<s<\infty$, (2.2) implies that the exponent of $\left|\psi^{\prime}(w)\right|$ in the above integral is negative. Also, since $\psi$ is a bounded schlicht function with $\psi(0)=0$, it follows that $\left|\psi^{\prime}(w)\right| \geqslant M\left(1-|w|^{2}\right)$ for all $w \in U$ for some positive constant $M$. Therefore

$$
\left\|\left(\phi^{\prime}\right)^{q / p}\right\|_{Q, p s}^{p s} \leqslant M_{1} \iint_{U}\left(1-|w|^{2}\right)^{4 s / \alpha-4 s-s q+2} d u d v,
$$

for another positive constant $M_{1}$. The last integral is finite if and only if (2.4) holds. This concludes the proof of the lemma. Note, however, that conditions (2.1), (2.2), and (2.4) are independent of $p(0<p<\infty)$.

3. Proof of Theorem 1'. The idea of the proof is to perturb $\alpha$ and $s$, subject to the restrictions of Lemma 4 , so that we have polynomial approximation in $\mathcal{F}_{\mathcal{Q}}^{p s}(D)$, and it suffices, according to Lemma 3 , to show that $\left(\phi^{\prime}\right)^{q / p}$ is in . $\mathcal{F}_{Q}^{p s}(D)$. The proof is done by successive perturbations. The result of Metzger [8] will be obtained as a special case of the first perturbation. Corresponding to Lemma 4 we let

$$
\begin{gathered}
\alpha_{n}=2 \frac{n+2}{n+4}, \quad n=0,1, \ldots ; \\
Q_{0}=2, \quad Q_{n}>1+\frac{1}{n+1}, \quad n=1,2, \ldots,
\end{gathered}
$$

and

$$
s_{n}>1, \quad\left(1-1 / \alpha_{n}\right) s_{n}=1-Q_{n} / 2, \quad n=0,1, \ldots
$$

Note that $s_{0}$ is free except, of course, that $s_{0}>1$. We now proceed by induction on $n$ to show that polynomials are dense in $\mathcal{F}_{q}^{p}(D)$ for all $0<p<\infty$ and all $q>1+1 /(n+2)$.

For $n=0, \alpha_{0}=1, Q_{0}=2$ and $s_{0}>1$. Using Lemma 1 we can assume that $1<q<2$. According to Lemma 1 the polynomials are dense in $\mathcal{H}_{Q_{0}}^{p s_{0}}(D)=$ $\mathcal{F}_{2}^{p s_{0}}(D)$. Using Lemma $4,\left(\phi^{\prime}\right)^{q / p}$ is in $\mathcal{F}_{2}^{p s_{0}}(D)$ if $s_{0}(q-1)>1$ and $s_{0} q<3$, that is, if $1 /(q-1)<s_{0}<3 / q$. Our assumption $1<q<2$ guarantees the existence of such $s_{0}>1$. Therefore $\left(\phi^{\prime}\right)^{q / p} \in \mathcal{F}_{2}^{p s_{0}}(D)$ if $q>3 / 2$ and it follows by Lemmas 3 and 4 that the polynomials are dense in $\mathcal{H}_{q}^{p}(D)$ for all $0<p<\infty$ and $q>3 / 2$. Assume now we have proved that the polynomials are dense in $\mathcal{H}_{q}^{p}(D)$ for all $0<p<\infty$ and all $q>1+1 /(k+1), k=$ 
$1,2, \ldots, n$. We shall show that this is true also for $q>1+1 /(n+2)$. Then we can assume that $1<q<2 / \alpha_{n}$ because $2 / \alpha_{n}=1+2 /(n+2)>1+$ $1 /(n+1)$. Since $Q_{n}>1+1 /(n+1)$ it follows from the induction hypothesis that the polynomials are dense in $\mathcal{F}_{Q_{n}}^{p s_{n}}(D)$. By Lemma $4,\left(\phi^{\prime}\right)^{q / p}$ is in $\mathcal{H}_{Q_{n}^{p}}^{p s_{n}}(D)$ if $s_{n}\left(1+q-2 / \alpha_{n}\right)>1$ and $s_{n}\left[q+4\left(1-1 / \alpha_{n}\right)\right]<3$; that is, if

$$
\frac{1}{1+q-2 / \alpha_{n}}<s_{n}<\frac{3}{q+4\left(1-1 / \alpha_{n}\right)},
$$

and our choice of $q\left(1<q<2 / \alpha_{n}\right)$ shows that (3.1) is contained in the range of $1<s_{n}<(n+2) /(n+1)$ if and only if $q>1+1 /(n+2)$ and then by Lemmas 3 and 4 the theorem follows.

4. Concluding remarks. We first note that Theorem 1 has the following

Corollary 1. Let $G$ be a Fuchsian group acting on $D$. Then the set of Poincaré series of polynomials is dense in $A_{q}^{1}(D, G), q>1$ (cf. Bers [1] and Knopp [7] for the appropriate formulation).

If we introduce the class $E^{p}(D)$ as, for example, in Duren [3, p. 168], then $E^{p}(U)=H^{p}(U), 0<p<\infty$. If $D$ is a bounded Jordan domain then $E^{p}(D)$ is a Fréchet space of holomorphic functions on $D$ normed by

$$
\|f\|_{p, D}^{p}=\operatorname{Sup}_{r<1} \int_{\gamma_{r}}|f(z)|^{p}|d z|
$$

where $\gamma_{r}$ is the image under $\psi$ of the circle $|w|=r$. Since $A_{q}^{p}(D)$ and $E^{1 / q}(D)$ are preserved under the same isometry induced by conformal mappings, it follows immediately from (1.3) that $E^{1 / q}(D) \subset A_{q}^{p}(D), 0<p<\infty, q p>1$. Using Theorem 1, we obtain

Corollary 2. $E^{1 / q}(D)$ is dense in $A_{q}^{p}(D), 0<p<\infty, q p>1$.

\section{BIBLIOGRAPHY}

1. L. Bers, Automorphic forms and Poincaré series for infinitely generated Fuchsian groups, Amer. J. Math. 87 (1965), 196-214. MR 30 \#4937.

2. Acta Math. 116 (1966), 113-134. MR 33 \#273.

3. P. L. Duren, Theory of $H^{p}$ spaces, Pure and Appl. Math., vol. 38, Academic Press, New York and London, 1970. MR 42 \#3552.

4. C. J. Earle and A. Marden, Projections to automorphic functions, Proc. Amer. Math. Soc. 19 (1968), 274-278. MR 37 \#12.

5. G. H. Hardy and J. E. Littlewood, Some properties of fractional integrals. II, Math. Z. 34 (1931), 403-439.

6. L. I. Hedberg, Weighted mean square approximation in plane regions, and generators of an algebra of analytic functions, Ark. Mat. 5 (1965), 541-552. MR 36 \#2808.

7. M. I. Knopp, $A$ corona theorem for automorphic forms and related results, Amer. J. Math. 91 (1969), 599-618. MR 40 \#4450.

8. T. A. Metzger, On polynomial approximation in $A_{q}(D)$, Proc. Amer. Math. Soc. 37 (1973), 468-470. MR 46 \#9361.

9. , On polynomial density in $A_{q}(D)$, Proc. Amer. Math. Soc. 44 (1974), 326-330. MR $49 \# 5375$. 
10. M. Sheingorn, Poincaré series of polynomials bounded away from zero on fundamental region, Amer. J. Math. 95 (1973), 729-749. MR 49 \#9194.

11. J. L. Walsh, Interpolation and approximation by rational functions in the complex domain, Amer. Math. Soc. Colloq. Publ., vol. 20, Amer. Math. Soc., Providence, R. I., 1935.

Department of Mathematics, Pennsylvania State University, University Park, PennSYLVANIA 16802

Department of Mathematics, University of Pittsburgh, Pittsburgh, Pennsylvania 15260 